Meta

Journal des traducteurs

Translators' Journal

\title{
Le traducteur canadien face au dictionnaire bilingue
}

\section{Jean-Paul Vinay}

Volume 18, numéro 1-2, mars 1973

Actes du deuxième colloque international de linguistique et de traduction. Montréal, 4-7 octobre 1972

URI : https://id.erudit.org/iderudit/004571ar

DOI : https://doi.org/10.7202/004571ar

Aller au sommaire du numéro

Éditeur(s)

Les Presses de l'Université de Montréal

ISSN

0026-0452 (imprimé)

1492-1421 (numérique)

Découvrir la revue

Citer cet article

Vinay, J.-P. (1973). Le traducteur canadien face au dictionnaire bilingue. Meta, 18(1-2), 179-188. https://doi.org/10.7202/004571ar d'utilisation que vous pouvez consulter en ligne.

https://apropos.erudit.org/fr/usagers/politique-dutilisation/ 


\section{Le traducteur canadien face au dictionnaire bilingue}

0. L'insertion de données lexicographiques canadiennes dans un dictionnaire monolingue ou bilingue est une décision importante pour le public francophone d'Amérique du Nord, ainsi que - à un moindre degré - pour le public anglophone. On sait que pareille démarche est assez récente ${ }^{1}$, suffisamment en tout cas pour soulever encore des polémiques et mériter quelques instants de réflexion. Au cours de la discussion qui a suivi le présent exposé, un participant s'est levé pour demander quel dictionnaire bilingue un traducteur canadien devait utiliser. À cette question très complexe je ne pouvais décemment que me dérober par une pirouette. J'ai répondu «Mais..., le mien, naturellement!» Mais au fond, en quoi le C.D.1 (et bientôt le C.D.2) seraient-ils préférables aux tomes de mes illustres confrères, qui font gémir de leurs poids les rayons de nos bibliothèques ? Réponse (et phrase) quelque peu ambiguës, mais problème très réel, dont je voudrais traiter ici rapidement.

\section{UN PEU D'HISTOIRE}

En 1955, sous l'impulsion de l'actif directeur de la firme McClelland \& Stewart Ltd., Toronto, il s'est créé à l'Université de Montréal un Centre de recherches lexicographiques ${ }^{2}$ dont la direction m'avait été confiée. Ce Centre qui faisait en quelque sorte partie intégrante de la Section de linguistique et de traduction (comme on disait alors) fit appel à des collaborateurs de l'extérieur (P. Daviault, H. Alexander, W. R. Jeanes, J. Darbelnet, B. H. Smeaton, W. F. Mackey, J. Rousseau) et utilisa les services de jeunes chercheurs qui ont depuis fait carrière en linguistique : G. R. Lefebvre, G. des Marchais, Lowell Williams. De nombreux étudiants montréalais ont fait également bénéficier nos fiches de leur connaissance du français québécois ou de l'anglo-canadien. Entre 1955 et 1958, certaines enquêtes ont été lancées, notamment sur la prononciation de l'anglo-canadien (D. E. Hamilton) et sur la normalisation de l'orthographe

1. Il n'est pas possible d'entrer ici dans une discussion sur la normalisation des dictionnaires. C'est là un problème très vaste et que tout lexicographe doit aborder plutôt tôt que tard. Il touche aussi bien à la phonétique : C.E. slough [slu :] ou [slau], C.F. mai [me] ou [me] ? - à l'orthographe : C.E. busses ou buses, C.F. Terreneuve ou Terre-Neuve? à la syntaxe : C.F. je l'aidais ou je lui aidais? - ou au lexique, auquel on pense de préférence, cf. Office de la langue française, Canadianismes de bon aloi : a ... seules sont acceptables les divergences qui se rapportent à des faits de vocabulaire.

2. Cf. Journal des traducteurs, "La lexicographie au Canada », I, 3 (juillet-septembre 1958) et II, 3-4 (janvier-mars 1959), en partiulier les articles de $\mathbf{P}$. Daviault, $\mathbf{M}$. $\mathbf{H}$. Scargifl et J.-P. Vinay. 
anglaise au Canada (F. E. Priestly). En 1958, devant la nécessité de récupérer une partie des frais encourus, et malgré l'aide financière de la Fondation Biermans, il a fallu se résoudre à ne préparer pour la publication qu'un dictionnaire «abrégé », qui est sorti des presses de Hazell, Watson \& Viney au début de 1962, sous l'égide de l'Université de Montréal : Dictionnaire canadien/The Canadian. Dictionary, XXXxiv-862 p., abrégé en C.D.1.

1.1. En 1970, la Donner Canadian Foundation m'a proposé de former une nouvelle équipe pour préparer une version plus élaborée du C.D.I, qui bénéficierait des nombreux travaux effectués récemment dans le domaine de la lexicologie et de la linguistique descriptive. Sous l'égide de l'Université de Victoria (C.B.), j'ai formé une petite équipe (M. T. Wilton, G. H. Hickman, C. Carey, F. Morgan) chargée de reprendre les données des enquêtes de 1955, de dépouiller systématiquement certains journaux canadiens (le Devoir, The Globe and Mail, The Victoria Daily Times), américains (The New York Times) et européens (le Monde, le Figaro, The London Daily Times), et de consulter les travaux canadiens récents, notamment ceux de l'Office de la langue française (Québec) et les fiches du Lexicographic Centre for Canadian English, que dirige à Victoria même mon collègue M. H. Scargill. Un traitement électronique simple a été prévu, basé en grande partie sur les travaux effectués pour Lexautom ${ }^{3}$, mais pour l'instant les fiches sont traitées manuellement, en prenant certaines précautions qui réservent l'avenir, telles que la confection de «séries» sémantiques limitées, conservées à part et constamment rapprochées de «séries» anglaises correspondantes ${ }^{4}$. $\mathrm{Ce}$ projet est désigné par l'abréviation C.D.2.

\section{DE QUEL DICTIONNAIRE S'AGIT-IL ICI ?}

La présente discussion intéresse plusieurs démarches également valables, mais qui appellent des solutions lexicographiques différentes. La majorité des dictionnaires modernes du français sont à sens unique (one-way), c'est-à-dire qu'ils définissent les termes d'une langue donnée en utilisant cette même langue pour le faire. Dans la mesure où ils réussissent à éliminer toutes considérations régionales ou dialectales, ils décrivent un usage relativement homogène. Pour désigner cet usage, il est donc inutile de recourir à des abréviations : la «Préface 》 doit suffire à le préciser et le titre y fait parfois allusion (cf. Dictionnaire du français contemporain).

3. Cf. Actes du [premier] Colloque international de linguistique et de traduction, numéro spécial, META XVI, 1-2 (mars-juin 1971), p. 5-131; voir aussi J.-P. Vinay et B. Kallio, Bilingual Lexicography and the Computer, compte rendu de la première phase du projet Lexautom, University of Victoria, 1971; M. T. Wilton, Computerized Bilingual Lexicography, Department of Linguistics, University of Victoria, 1972, inédit.

4. Par série lexicologique, $\mathrm{j}$ 'entends ces sous-ensembles au sein desquels se nouent des oppositions, et dont l'exploration justifie l'existence des subdivisions sémantiques des articles du dictionnaire. Par exemple bleu rentre dans la série couleurs, où il s'oppose à rouge, vert, etc. ; il rentre aussi dans la série politique, où il s'oppose à rouge, péquiste, etc. ; il rentre enfin dans la série états d'âme, où il s'oppose à (avoir du) fun, etc. Ceci n'est pas vrai en F.F., où bleu n'est guère employé au sens politique que dans des expressions archaïsantes (bas bleu). Par contre, j'en suis (resté) bleu rentrerait dans la série états d'âme, et bleum. figure dans la série classement social au titre militaire, et dans la série vêtements (de travail) : un bleu (de chauffe). La question est de savoir si ces trois derniers sens existent également en C.F. 
2.1. Mais il est rare que les lexicologues n'aient pas à considérer plusieurs variétés d'un même usage. Sans comporter toujours de valeur péjorative, ces variétés sont généralement distinguées par une étiquette, abrégée d'une façon ou d'une autre. La gamme en est très vaste, depuis le français populaire (F.Pop.) jusqu'aux régionalismes, qui comprennent depuis quelque temps des canadianismes (F.C. ou C.F.), des belgicismes (F.B.), etc., qu'on ne trouvait auparavant que dans des glossaires spécialisés. $\mathrm{Si}$ cette référence à des usages parallèles est constante et systématique, on a vraiment affaire à un dictionnaire bilingue (deux usages) ou multilingue (multiples usages). Cependant ces étiquettes ne s'emploient guère, bien qu'en fait elles soient parfaitement justifiées. La raison en est double : a) les variétés du français ou de l'anglais sont rarement considérées comme constituant des "langues » séparées ${ }^{5} ; b$ ) les dictionnaires de ce type, comme nous le verrons plus bas, n'envisagent le plus souvent qu'un très petit nombre de régionalismes ou de formes dialectales, et demeurent au fond à sens unique.

2.2. En fait, cette façon de procéder, qui représente des avantages certains, doit s'entendre comme la rationalisation d'un état de choses beaucoup plus complexe. J'ai eu récemment l'occasion d'évoquer dans un article encyclopédique ${ }^{6}$ la subdivision généralement admise des dialectes et sous-dialectes du français en Amérique du Nord (F.A.N.), que l'on peut schématiser de la façon suivante :

\section{FIGURE 1}

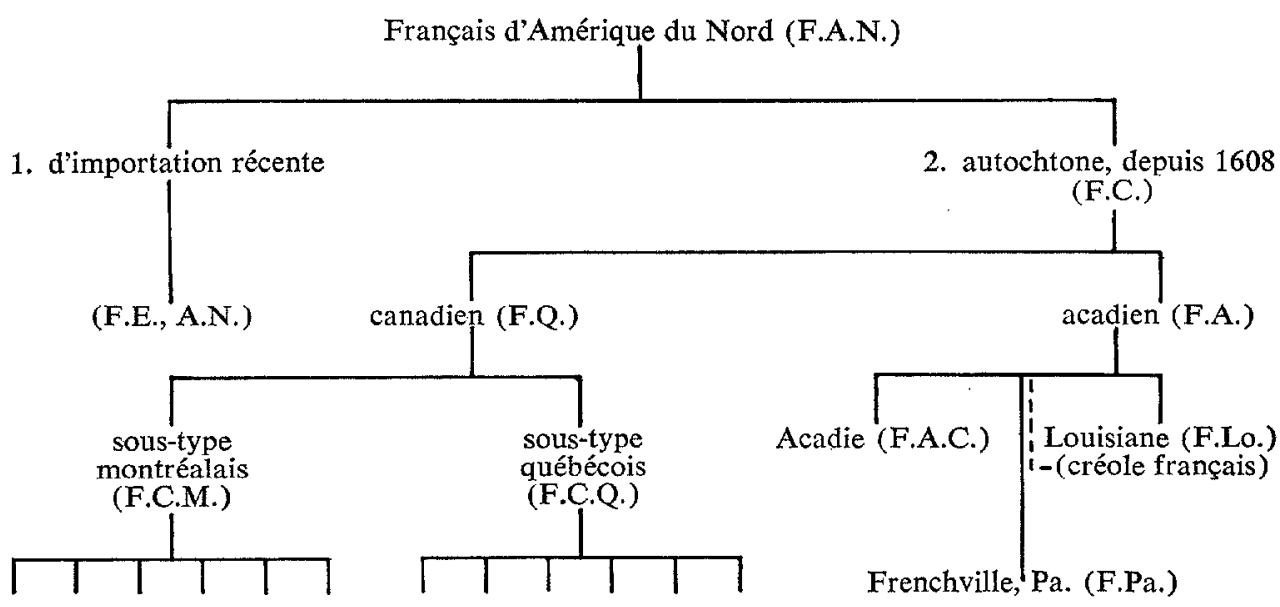

variétés régionales suivant

les provinces ou les Etats

américains (F.Ont., F.Man., F.Mass.)

5. On dit cependant constamment "traduit de l'américain », ce qui semble reconnaître une certaine autonomie linguistique au pays que les Français s'obstinent à appeler a les U.S.A.». Il ne semble pas que ce soit le cas pour les livres suisses ou belges. Mais avec les formes extrêmes du "québécois ", on entrevoit la possibilité d'une traduction; ceci a lieu, me dit-on, pour certains films québécois projetés en France en version originale " avec sous-titres en français ".

6. "Le Français en Amérique du Nord : problèmes et réalisations ", in T. Sebeok, Current Trends in Linguistics II, La Haye, Mouton (sous-presse). 
Ce schéma montre bien que le «québécois » dont on parle tant en ce moment (F.Q.) est la somme de plusieurs sous-dialectes, qui peuvent se grouper assez différemment selon le cas, et qu'en utilisant dans nos dictionnaires des sigles d'attribution du type C. ou F., on se place en fait à un haut niveau d'abstraction. Il convient done de lire ces sigles comme suit :

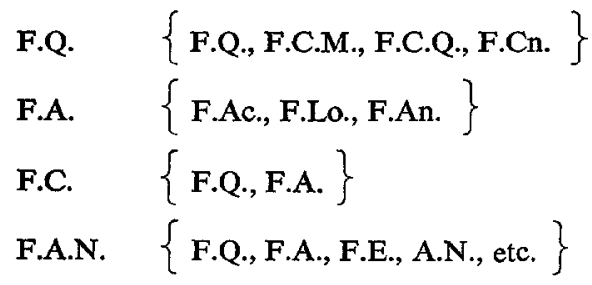

Ces considérations ne sont pas envisagées pour compliquer les choses à plaisir. Il se peut fort bien que l'état actuel de nos connaissances en matière de régionalismes ne soit pas assez avancé pour dire si vraiment bourgot est F.A.N. ou seulement F.Q. ou même uniquement F.C.Q.; il serait pourtant utile de pouvoir préciser l'extension géographique ${ }^{7}$ de termes comme aboiteau, barachois, postillon, ou, pour l'anglais, de slough, sockeye, reeve, concession road, etc.

\section{LE CAS DES DICTIONNAIRES BILINGUES}

Nous avons vu qu'un dictionnaire rédigé uniquement en français, mais reflétant diverses variétés régionales, voire dialectes, est une sorte d'ouvrage bilingue. Il semble pourtant que cette étiquette soit réservée au rapprochement de deux langues «à part entière»; c'est le cas de la majorité des dictionnaires auxquels les traducteurs ont l'occasion de faire appel : Dictionnaire français-ouolof, français-roumain, français-latin, et bien entendu français-anglais. En théorie, il ne semble pas utile de préciser de quelle langue il s'agit, la disposition typographique, ou certaines marques particulières pouvant suffire. Par exemple, une entrée telle que fork, fourchette est claire en elle-même, mais s'éclairera d'autant plus quand on la trouve sous la forme fork, $n$., fourchette $f$. Cela permet de distinguer parfaitement des homographes tels que : telephone, $n$., téléphone ${ }^{m}$; journal, n.m., journal. Mais là aussi, il est rare que les dictionnaires ne donnent pas des exemples de régionalismes, par exemple des américanismes au sein d'un ouvrage ouvertement britannique tel que le Harrap ${ }^{8}$. Des abréviations telles que Brit. ou U.S. sont fréquentes dans ces dictionnaires.

3.1. Un dictionnaire qui se veut «canadien» devra donc citer plusieurs usages régionaux ou nationaux, et devenir à la fois «bilingue » et «multilingue ». En simplifiant, il devra tenir compte au moins de deux usages français, celui de France et celui du Canada, et trois usages anglais, celui de la Grande Bretagne, du Canada et des États-Unis, qui pourraient s'abréger en F.F. et F.C., E.Br., E.C.

7. Sans parler, bien entendu, de leur valeur stylistique ou de leur niveau linguistique.

8. Une remarque, en passant. Pourquoi parle-t-on généralement « du Harrap's ", ailors qu'on parle « du Duden ", « du Webster " ou " du Larousse " ? La terminaison 's ne s'impose pas en français, sauf pour les snobs parisiens qui fréquentent le Fouquet's. Il serait d'ailleurs plus exact de parier * du Mansion", comme on parle " du Robert", ou à la rigueur « du Ledésert " comme on pourrait parler * du Rey ». 
et E.U.S. Pour simplifier, on pourrait écrire simplement F., C., C., U.S., ce qui crée cependant une ambiguité que pourra lever la disposition typographique.

Ex. C. gros char(s) n.m.pl. [ = F. chemin ${ }^{m}$ de fer $]$

C. [Br.] railway [U.S. railroad].

en admettant que la réalité se présente bien réellement ainsi.

3.2. On peut évidemment n'utiliser qu'un seul sigle pour tout ce qui est senti comme canadien, par exemple $q_{0}, *$ ou (C). Mais il ne faut pas oublier que le lecteur a besoin de savoir, pour chaque langue, l'extension géographique des mots proposés et en particulier si un terme anglais préfixé de (C) est rendu dans le dictionnaire par un terme qui se situe au même niveau géographique. Dire par exemple que (C) terme anglais $x=$ terme français $y$ n'est pas suffisant; il faut préciser si le second terme est uniquement F.C. ou également F.F. Par exemple :

- stove $n .=$ (C) poêle ${ }^{m}$., (F.) fourneau ${ }^{m}$, cuisinière $\%$.

- camion n.m. = (C) truck, (Br.) lorry.

Les mots pinte et pint sont de bons exemples d'ambiguïtés différentes selon la langue de départ qu'il convient de dissiper :

(C) pinte $n . f .[\mathrm{F}=$ environ un litre], quart $[=2$ pints. N.B. : il existe des variations locales C.E., Br. et U.S. de la valeur exacte de cette mesure].

(F.) pinte n.f. [ô a good time.

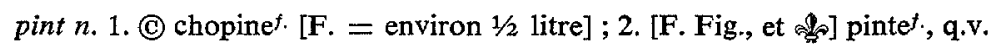

Il fut un temps où moose n'était représenté dans les dictionnaires français que par élan d'Amérique ; depuis quelque temps, le mot orignal (considéré comme (C) a fait son apparition dans ces mêmes dictionnaires. Il y a là deux usages, dont le premier est très justifié hors du Canada (de même que l'expression pain français hors de France, et assiette anglaise hors des pays britanniques) et que l'on pourrait représenter comme suit :

(C) moose [ ?U.S., ??Br.] $n .=$ (C) orignal ${ }^{m .}$, (F.) élan ${ }^{m .}$ d'Amérique, $*$ orignal $^{m . ~}{ }^{9}$.

\section{QU'EST-CE DONC QU'UN CANADIANISME ?}

Parler de F.C., de F.Q. ou de E.C. pose évidemment la question - toujours brûlante - de la définition d'un canadianisme, ou de toute variété de langue ${ }^{10}$.

9. Les points d'interrogation dénotent de ma part une ignorance quant à l'origine et l'extension du mot. Ils n'apparaîtraient sans doute pas dans l'édition définitive. L'astérisque indique un néologisme ou un emprunt.

10. La question des "variétés géographiques d'une koïné ou langue commune 》 a fait l'objet de plusieurs études récentes, notamment l'essai de définition de M. Lothar Wolf, paru dans les Travaux de linguistique et de littérature de Strasbourg, vol. $\mathrm{X}, \mathrm{n}^{\circ} \mathrm{1}, 1972$, p. 171-177. Mais elle demeure encore floue, lorsqu'il s'agit de définir ces variétés « par rapport à leur situation en face d'une norme officielle qu'on a appelée norme commune, français normal, français général, français standard, etc., et qui fixe l'usage linguistique des milieux cultivés, notamment de la capitale $₫$. Je ne désire pas entamer ici une discussion sur la validité des faits linguistiques régionaux par rapport à une norme : pour ceux qui sont curieux d'histoire, Mencken (The American Language, I) a brossé un tableau intéressant des réactions britanniques et américaines à l'usage de formes américaines depuis le début de l'époque coloniale. Il y a là un parallélisme intéressant avec les faits québécois et la longue querelle du purisme dont Guy Bouthillier et Jean Meynaud viennent de donner une imposante anthologie (le Choc des langues au 
Au moment où un nouveau C.D.2 est en voie de préparation, cette question demande un nouvel examen et, pour le lexicologue, elle n'est pas académique. L'emploi de plusieurs schémas emprunté aux «mathématiques nouvelles » pourra éclairer les véritables données du problème.

4.1. Une langue considêrée comme parfaitement homogène pourrait être représentée comme un ensemble de termes qui se définissent par opposition les uns aux autres (fig. $2 \mathrm{X}$ ).

\section{FIGURE 2}
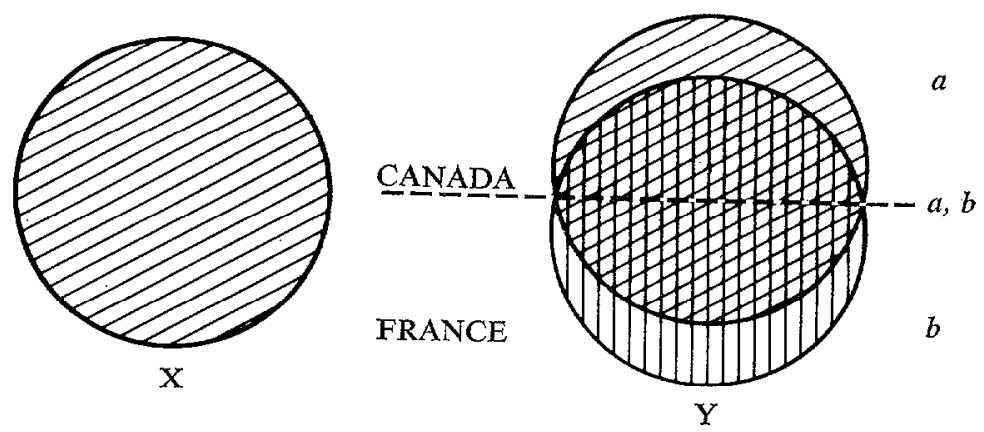

Par contre, une langue qui possède une forme régionale ou dialectale peut représenter une distribution de ses éléments beaucoup plus complexe. Certains termes ne seront utilisés ou compris que dans une région donnée (fig. $2 \mathrm{Y}: a$ ou $b$ ), d'autres au contraire seront employés de façon plus ou moins identique dans toutes les régions (fig. $2 \mathrm{Y}: a, b$ ).

Si l'on applique ce schéma au français, on voit que certains termes utilisés exclusivement dans la zone $a$ devront être expliqués pour les locuteurs de la zone $b$, de même que ceux de la zone $b$ pour la zone $a$; le reste des termes étant en principe également accessible aux locuteurs des zones $a$ et $b$. On pourrait désigner $a$ et $b$ des zones marginales et $a, b$ la zone centrale (en anglais core).

4.2. Si l'on examine maintenant quelques définitions du terme «canadianisme», on s'aperçoit qu'elles recouvrent en général la zone marginale $a$, mais qu'elles excluent les termes de la zone centrale $a, b$. Les définitions se compliquent aussi de considérations normatives (canadianismes de «bon» ou de «mauvais aloi »), stylistiques et historiques.

4.2.1. La page titre du Glossaire de la Société du parler français au Canada (Québec, 1930; réimpression 1968) indique nettement que cet ouvrage ne s'intéresse qu'aux mots et locutions en usage dans le parler de la province de Québec et qui ne sont pas admis dans le français d'école. Il ne considère donc en principe que les mots de $a$, sans d'ailleurs être complet. Il ne dit rien par exemple

Québec, 1760-1970, Montréal, Les Presses de l'Université du Québec, 1972, 770 p.). Voir aussi A. Viatte, la Francophonie, Paris, Larousse, 1969 ; S. Begtsson, la Défense organisée de la langue française, Uppsala, Acta Universitatis Upsaliensis 1968 ; et dans un autre ordre d'idées, J. O. Granjouan, les Linguicides, Didier international, 1971. 
de bleutière, orignal, citron, brûlot, pied-de-Roi, acre et il explique demiard, ferrer, mauvais matin, flâner et même... football, qui sont au moins connus en $a, b$.

4.2.2. La préface canadienne du dictionnaire de Cassell, due à la plume de Gaston Dulong, explique son choix de certains « canadianismes de vocabulaire » qui caractérisent uniquement la zone $a$ :

Seuls ont été retenus les mots (substantifs, adjectifs ou verbes) que j'appellerai canadianismes de vocabulaire. Ce sont des mots concernant des réalités proprement canadiennes, pour lesquels le français commun, né sous un autre climat et d'autres latitudes, n'a pas d'équivalent. Ces canadianismes ont trait surtout à l'hiver, à la faune, à la flore, aux modes de vie, aux habitudes. Autant" de domaines pour lesquels les francophones du Canada ont dû créer on adapter. Encore fallait-il que ces mots fussent d'un usage assez fréquent pour mériter de figurer dans un dictionnaire du genre de Cassell's.

4.2.3. Par contre, les auteurs du Dictionary of Canadianisms Based on Historical Principles élargissent beaucoup leur définition des canadianismes, couvrant à la fois les zones $a$ et $a, b$ :

Since our approach to our material is clearly quite different from that of lexicographers in the United States, we have chosen to define Canadianism less exclusively than they have defined Americanism. A Canadianism, then, is a word, expression or meaning which is native to Canada or which is distinctively characteristic of Canadian usage though not necessarily exclusive to Canada; Winnipeg couch falls into the first category, Chesterfield ( $\ll$ sof $a »$ ) into the second.

4.2.4. Le C.D.1 enfin essaie d'élargir encore plus la définition en considérant qu'un usager canadien a besoin de connaître aussi bien les termes a que les termes $b$, et que la majorité de ses problèmes de définition et de traduction se situe probablement en $a, b$. Cette attitude est reflétée clairement par les notes liminaires (p. xxix) :

Tous les articles dont la langue de départ est l'anglais reflètent l'usage canadien et nord-américain à moins d'indication contraire. Leur traduction reflète l'usage commun au franco-canadien et au français international. Les signes (C) ou [Fr.] précisent les mots ou expressions d'usage exclusivement canadien ou européen.

Pour un mot tel que potatoes cela donne :

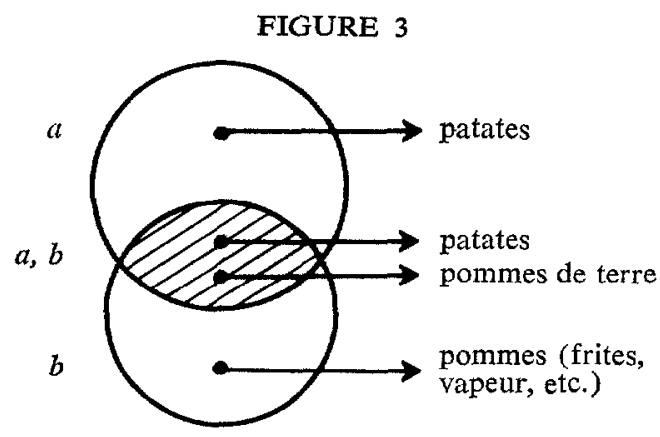

Ce qui revient à dire que la zone centrale ou core est absolument essentielle pour s'assurer si les différents sens d'un même mot sont représentés ou non dans les zones marginales. On pourra étudier ainsi la répartition des sens de termes 
aussi communs que bleu, chaud, fin, entre les zones $a, a, b$ et $b$. Il est plus que probable que le sens politique de (C) bleu, le sens mélioratif de (C) fin et le sens péjoratif de (C) chaud n'existent pas en $a, b$. Ce sont vraiment des canadianismes. La figure 4 représente un essai de classement de quelques termes, dont certains doivent apparaître plusieurs fois selon le sens considéré :

\section{FIGURE 4}

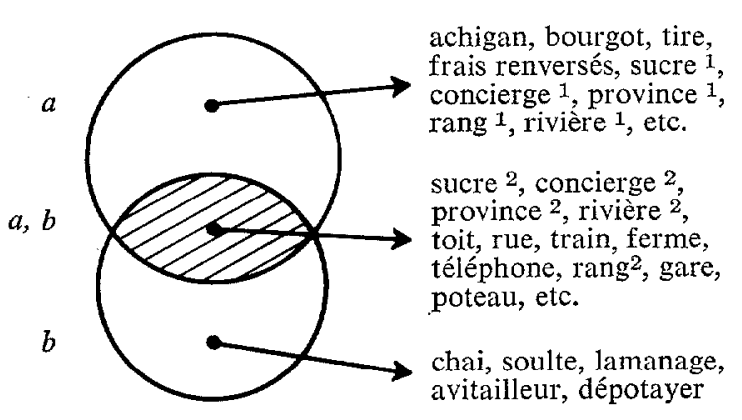

\section{CONCLUSION}

La présente communication se veut donc un plaidoyer pour une lexicographie intégrée, reflétant parfaitement la réalité canadienne dont elle note, dans une optique comparative, tous les aspects sémantiques et stylistiques. C'est là bien entendu un idéal, et la complexité d'une langue en pleine évolution ne doit pas décourager les chercheurs. Au Canada plus que partout ailleurs dans le monde francophone, la langue est un devenir, et tout dictionnaire est de dix ans en retard le jour de sa publication. Il n'en reste pas moins que le «placage » trop facile d'un échantillonnage de termes canadiens sur un corpus français passe-partout trahit le lecteur, qu'il soit traducteur, rédacteur ou « honnête homme ». Pour remplir utilement son rôle, un dictionnaire bilingue canadien doit refléter au moins cinq usages différents, essayant constamment de rapprocher C.F. et F.F. de C.E., U.S., Br. Les frontières linguistiques ne doivent pas passer à côté des dictionnaires : elles les traversent de part en part.

5.1. Ceci dit, on peut noter quelques conséquences qui se dégagent de ces prémisses. Il me paraît notamment qu'il existe trois limites à la démarche multilingue définie plus haut :

1) Des limites théoriques, qui dépendent des possibilités d'enquête dont dispose l'équipe de rédaction. Dans un récent article du Victoria Daily Times (23 février 1973), le $D^{r} \mathrm{D}$. Leechman décrit ses efforts pour relever des termes canadiens qui pourraient figurer au dictionnaire d'Oxford. Il cite en particulier housekeeping cabins, dont il a trouvé un exemple dans le Globe and Mail. Première faiblesse du système : il aurait pu passer à côté de cette citation, ou ne pas reconnaître le caractère canadien du terme. Mais une fois trouvée la définition de housekeeping cabins : $A$ holiday cabin or chalet in which the occupants are 
expected to do their own housekeeping », reste à savoir si le terme est U.S. (ou s'il existe un équivalent U.S.) et à quoi il correspond en $\mathrm{Br}$. Si le $\mathrm{D}^{\mathrm{r}}$ Leechman travaillait sur un dictionnaire bilingue il lui faudrait encore chercher l'équivalent C.F. d'une donnée culturelle qui existe certainement au Québec pour l'équivalent F.F. (néologisme ou emprunt) en usage en Europe. On aura noté que le lexicologue bilingue ne donne pas (en général) de définition (fig. $2 \mathrm{X}$ : a) mais rapproche les termes équivalents (fig. $2 \mathrm{X}: \mathrm{b}$ ).

a) Terme nouveau $\longleftrightarrow$ Définition

b) Terme nouveau (Définition) $\rightarrow$ Terme(s) équivalent(s)

Cette difficulté pourrait être résolue par des notes donnant simplement les éléments de la définition. Il s'agirait surtout de ces cas limites dits « intraduisibles », pour lesquels le lecteur a besoin de renseignements lui permettant de comprendre sans traduire. Un exemple : pour décrire l'état d'esprit d'un de ses héros, Nevil Shute emploie l'expression «Been missing your Kruschens again?» Le roman date de 1923 ; à cette époque les sels Kruschen faisaient une publicité tapageuse basée sur un personnage en pyjama exhibant un sourire éclatant. Il faut donc traduire la remarque par un équivalent, et faute de mieux je suggère \& Faut faire une cure à Vichy» ou encore «Prenez des pilules Pink» ou encore «Votre foie ne tourne pas rond ». Mais il est peu probable que ces suggestions puissent figurer dans un dictionnaire, qui se contenterait d'expliquer l'allusion ${ }^{11}$. On doit noter en passant que l'un des éléments de ces limites théoriques est la compétence de l'usager, qui doit - idéalement - être sensibilisé à tous ces usages et à tous ces niveaux de langue.

2) Des limites typographiques; la multiplication des sigles indispensables était parfois source de confusion pour le lecteur. Le nombre des sigles n'est pas infini, et le recours à l'ordinateur, si souhaitable en matière de lexicographie, limite encore les possibilités. C'est ici que se placeraient les considérations de traitement de l'information abordées dans les rapports de Lexautom (Victoria), de Termium (Montréal, Banque de terminologie) ou de Baie Saint-Paul (O.L.F.Q.). Mais ceci est une autre histoire.

3) Des limites financières, que l'on imagine facilement, aussi bien du côté rédaction (multiplication des équipes de recherche) que du côté production (coût de la composition du manuscrit).

Mais la conséquence la plus importante des considérations précédantes se trouve, me semble-t-il, dans cet effort de synthèse qu'appelle une conception élargie des canadianismes (gallicismes, et autres américanismes), aboutissant en fait à la création d'un dictionnaire plurinational et pluriculturel sans lequel il est impossible de rendre pleinement compte de la complexité d'un texte.

Jean-Paul Vinay

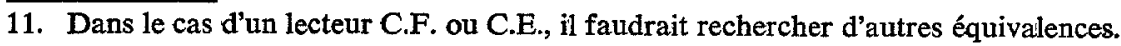




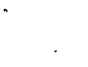

